An official journal of the / Un journal officiel de la "Société Sénégalaise de Cancérologie" (SOSECAN)

Journal homepage: www.africanjournalofoncology.com

Original article / Article original

DOI: https://www.doi.org/I0.54266/ajo.I.2.45.NROO2456

\title{
Giant uterine leiomyoma delivered through the cervix: About three cases in Ouagadougou
}

\section{Léiomyome utérin géant accouché par le col : A propos de trois cas à Ouagadougou}

\author{
N. Zongo', AH. Bagué2, M. Windsouri', PB. Yaméogo', NML. Ouédraogo', J. Sawadogo'.
}

I Centre hospitalo-universitaire Yalgado Ouédraogo de Ouagadougou, Burkina Faso.

2 Pôle chirurgie du centre hospitalier régional de Kaya, Burkina Faso.

${ }^{3}$ Service de chirurgie, CHU Tengandogo, Burkina Faso.

INTRODUCTION: Giant uterine leimyofibromas delivered through the uterine cervix are rare. MATERIALS AND METHODS: We report 3 observations in order to describe our diagnostic and therapeutic approach. RESULTS: Three patients aged 40, 49 and 4I years were seen for intense pelvic pain associated with metrorrhagia and a feeling of pelvic heaviness. The average delay of consultation was three years. They were all multiparous. The gynecological examination revealed a firm tissue mass with a smooth and regular surface of eight $\mathrm{cm}$ on average, occupying the vaginal cavity while respecting the vaginal walls. Speculum examination was not possible because of the size of the mass which was flush with the lower third of the vagina. Magnetic resonance imaging concluded to a myoma delivered through the cervix in all patients. A total hysterectomy was performed. Histology of the total hysterectomy specimen concluded a uterine leiomyoma of the uterus. CONCLUSION: These giant uterine leiomyomas are rare. Magnetic resonance imaging describes them well. The treatment is most often a total hysterectomy in women who do not wish to have pregnant.

KEYWORDS: Giant uterine myomas; MRI; Hysterectomy.

INTRODUCTION : Les léimyofibromes utérins géants accouchés par le col utérin sont rares. MATERIELS ET METHODES : Nous rapportons 3 observations dans le but de décrire notre approche diagnostique et thérapeutique. RESULTATS : II s'agit de trois patientes âgées de 40,49 et $4 \mathrm{I}$ ans reçues pour des douleurs pelviennes intenses associées à des métrorragies et à une sensation de pesanteur pelvienne. Le délai moyen de consultation était de trois ans. Elles étaient toutes multipares. L'examen gynécologique a permis de noter une masse tissulaire ferme, à surface lisse et régulière de huit $\mathrm{cm}$ en moyenne, occupant la cavité vaginale tout en respectant les parois vaginales. L'examen au speculum n'était pas possible à cause la taille de la masse qui affleure le tiers inférieur du vagin. L'imagerie par résonance magnétique a évoqué un myome accouché par le col chez toutes les trois patientes. Une hystérectomie totale a été réalisée chez toutes les patientes. L'histologie de la pièce d'hystérectomie totale a conclu à un léiomyome utérin dans tous les cas. CONCLUSION : Les léiomyomes utérins géants accouchés par le col sont rares. L'imagerie par résonnance magnétique est d'un apport notable dans le diagnostic. Le traitement est le plus souvent une hystérectomie totale chez les femmes non désireuses de grossesse.

MOTS-CLES : Myomes utérins géants ; IRM ; Hystérectomie.

\section{INTRODUCTION}

Les myomes utérins sont les tumeurs les plus fréquentes de l'utérus [I]. Ils représentent $70 \%$ des tumeurs bénignes de la femme [I, 2]. Ils surviennent chez les femmes âgées de 30 à 50 ans $[I, 2]$. Les symptômes les plus fréquents sont constitués par les douleurs et les saignements anormaux de l'utérus dont les hyperménorrhées et les métrorragies. Les facteurs de risque sont représentés par les ménarches précoces, la nulliparité et le phototype mélanoderme [I]. Plus de $96 \%$ des myomes utérins sont de localisation corporéale. Moins de I\% des localisations intéressent le col de l'utérus [I, 2]. Les myomes à localisation cervicale sont de survenue rare [2]. Dans la littérature africaine, les données sur la particularité de ces myomes sont rares et ils sont très souvent rapportés dans des études traitant des léiomyomes utérins en générale [3]. Le diagnostic pré-thérapeutique constitue parfois un défi du fait du polymorphisme clinique [4]. La chirurgie demeure le principal moyen thérapeutique de ces tumeurs utérines bénignes à topographie cervicale [2]. Nous rapportons une série de trois cas de léiomyomes géants accouchés par le col, dans le but de décrire notre approche diagnostique et thérapeutique.

\section{OBSERVATIONS}

Cas I

Mme N.C., 49 ans, femme au foyer a consulté en octobre 2015 pour des métrorragies abondantes. Le début de la symptomatologie remonterait à trois ans marqué par des douleurs pelviennes d'abord intermittentes puis permanentes, intenses et paroxystiques, rebelles aux antalgiques de palier II, requérant de la morphine. Elles étaient accompagnées de métrorragies faites de sang rouge, d'abondance croissante. Par ailleurs, des constipations et de la pollakiurie étaient rapportées. La patiente était multigeste (neuf fois), multipares (sept accouchements). Aucun antécédent personnel de myome ou de cancer, ni de comorbidité n'a été retrouvé. II n'y avait

* Corresponding author: Dr Nayi Zongo, Maître de Conférences Agrégé, Cancérologie Chirurgicale, CHU Yalgado Ouédraogo, Université Joseph KI-ZERBO, Burkina Faso. 
pas d'histoire familial de myome ou de cancer dans la famille. L'examen générale retrouvait une pâleur conjonctivale et palmo-plantaire, une pression artérielle à $100 / 60 \mathrm{mmHg}$ et un pouls à 95 pulsations/min. Une masse pelvienne peu mobile était retrouvée à la palpation hypogastrique. Au toucher vaginal, le doigt butait sur une masse comblant le vagin, ferme, à surface lisse non adhérant aux parois vaginales qu'elle refoule. Au toucher vaginal combiné au palper abdominal, la masse était mobile avec l'utérus. Au toucher rectal, la masse bombait dans le rectum qu'elle comprimait.

L'imagerie par résonnance magnétique (IRM) concluait à un volumineux myome accouché par le col de $15 \mathrm{~cm}$ de grand axe avec un utérus reposant sur le myome telle « la lanterne sur le dôme de l'église saint Paul de Londres » (Figure I). Une anémie avec un taux d'hémoglobine de $7 \mathrm{~g} / \mathrm{dl}$ a été objectivée par le bilan préopératoire et corrigé par une transfusion de sang Isogroupe-isorhésus. Elle a bénéficié d'une laparotomie médiane sous-ombilicale sous anesthésie générale. L'exploration chirurgicale a permis de retrouver des ovaires non tumoraux et un utérus dont le corps était indemne de tumeur et la partie cervicale siège de tumeur à développement endovaginal. II a été réalisé une hystérectomie totale (Figure 2). L'examen anatomopathologique de la pièce opératoire a conclu à un léiomyome cervical de l'utérus. Les suites opératoires ont été simples.

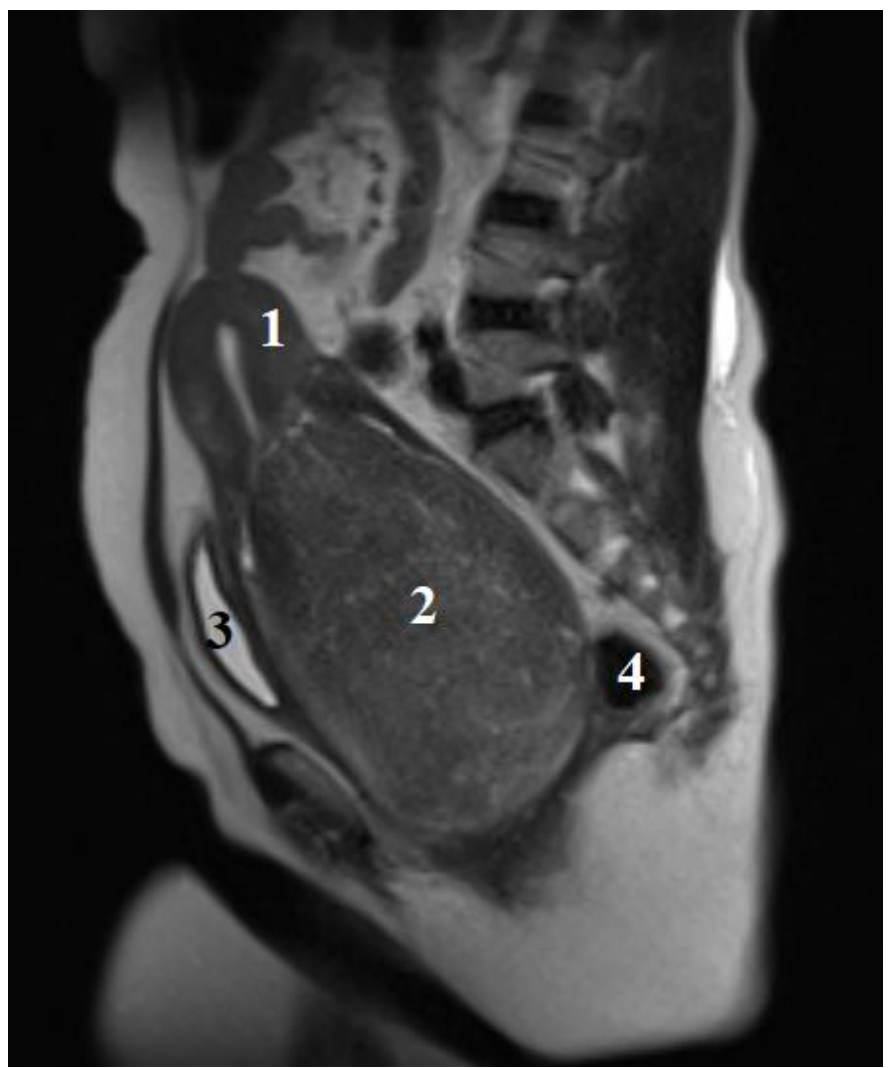

Figue I : Coupe sagittale d'IRM montrant le léiomyome accouché par le col occupant la cavité vaginale. I: Utérus, 2: Myome cervical, 3: Vessie, 4: Rectum.

\section{Cas 2}

Mme B.S., 40 ans, a consulté en Août 2018 pour des métrorragies évoluant depuis environs cinq ans. Il s'agissait de métrorragies d'abord d'abondance minime associées à des douleurs pelviennes, des constipations chroniques et une pollakiurie.

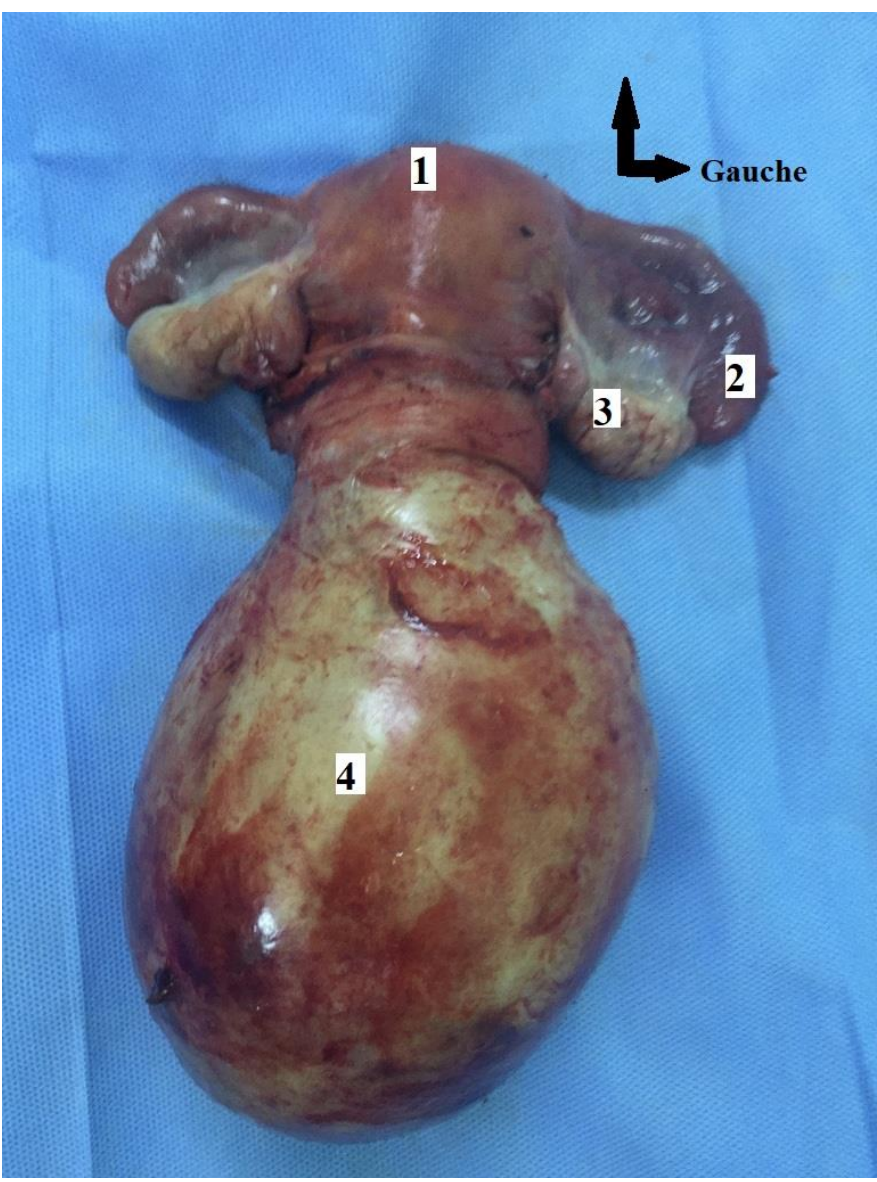

Figue 2 : Pièce opératoire d'hystérectomie et annexectomie bilatérale pour volumineux myome cervical : Aspect en «l lanterne sur le dôme de Saint Paul ». I: Utérus, 2 : Trompe droite, 3 : Ovaire droit, 4 : Myome cervical.

Après recours à un traitement auprès des tradi-praticiens sans succès, elle a consulté en milieu médical pour meilleure prise en charge. Dans ses antécédents gynécologiques, nous retrouvons une ménarche à 14 ans, trois grossesses et trois parités avec un cycle menstruel perturbé du fait des métrorragies. Elle n'avait pas d'antécédents médicochirurgicaux. Aucune histoire familiale de myome ou de cancer n'a été rapportée. A l'admission dans notre centre, elle avait un statut de performance selon l'OMS à 0 et des constantes normales. A l'examen physique, une masse hypogastrique dure, aux contours bien limités, indolore était palpée. Au toucher vaginal, le doigtier se heurtait à une masse dure aux limites nettes, à surface régulière et lisse, non adhérente à la paroi vaginale de sept $\mathrm{cm}$ de grand axe. Les culs-de-sac vaginaux n'était pas perçus. La masse hypogastrique était mobile lors de la mobilisation de la masse endovaginale au toucher vaginal combiné au palper abdominal. Le doigtier ramenait des traces de sang. La tumeur faisait effet de masse sur le rectum au toucher rectal. L'échographie pelvienne a retrouvé un gros utérus siège d'une masse cervico-isthmique peu vascularisée au Doppler. L'IRM notait une masse utérine cervico-isthmique hétérogène a développement endovaginal. Le traitement a été une hystérectomie totale. Les suites opératoires ont été simples.

Cas 3

Mme K.S., 4I ans, a consulté en Mai 202I pour des métrorragies et des douleurs pelviennes évoluant depuis plus d'un an. Elle n'avait pas d'antécédents de cancer dans la famille. 
L'examen clinique a permis de retrouver un statut de performance OMS I. II a été noté une pâleur palmo-plantaire et conjonctivale, une polypnée à 24 cycles/minutes, une tachychardie à 105 pulsations/minutes. La tension artérielle était de $90 / 60 \mathrm{mmHg}$.

Au toucher vaginal, la masse était arrondie avec une surface régulière et lisse. Elle comblait la lumière vaginale. Les parois vaginales étaient régulières. Les paramètres étaient souples. Le taux d'hémoglobine était de $3 \mathrm{~g} / \mathrm{dl}$. L'IRM a retrouvé un utérus siège de multiple masses, dont une cervico-isthmique à développement endocavitaire prolabant dans le vagin (Figure $3)$. La patiente a bénéficié d'une réanimation préopératoire, de transfusion de concentrés de culot globulaire isogroupeisorhésus. Ce qui a permis d'obtenir un taux d'hémoglobine à $10 \mathrm{~g} / \mathrm{dl}$. En peropératoire, l'exploration a mis en évidence un utérus polymyomateux avec le plus gros myome accouché par le col. II a été réalisé une hystérectomie totale avec annexectomie bilatérale (Figure 4). Les suites opératoires ont été simples.

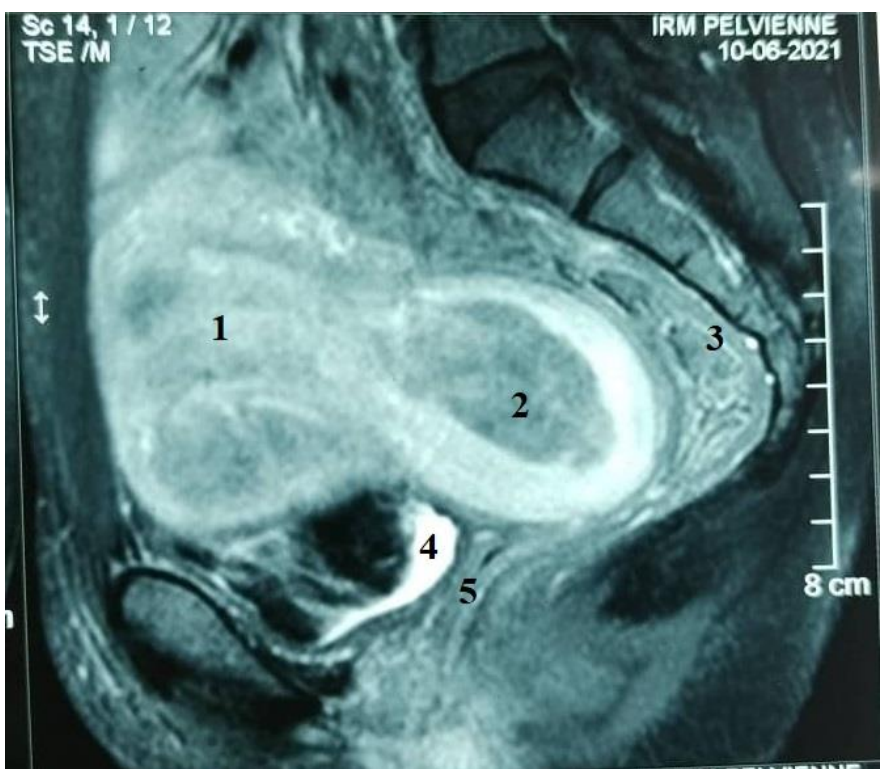

Figue 3 : Aspect IRM en phase T2 d'un utérus polymyomateux, dont un myome accouché par le col (coupe sagittale). I : Utérus polymyomateux, 2 : Myome accouché par le col, 3 : Rectum, 4 : Vessie, 5 : Vagin.

\section{DISCUSSION}

Les léiomyomes utérins, encore appelés «fibromes utérins » ou « myomes utérins », à localisation cervicale représentent une forme topographique rare de ces tumeurs gynécologiques les plus fréquentes $[1,2]$. Ils constituent moins de $2 \%$ des léiomyomes utérins [ $[, 2]$. La pauvreté en fibres musculaires lisses de la partie cervicale par rapport au corps de l'utérus explique en partie cette faible fréquence des léiomyomes cervicaux $[1,2]$. Les facteurs de risque de survenue restent cependant similaires aux léiomyomes utérins en général [I, 2]. Ce sont les facteurs concourant à une hyperoestrogénie (ménarche précoces, nulliparité), les conditions ethnogénétiques (mutation du gène MEDI2, femme noire) et les troubles métaboliques (obésité) [3, 5, 6]. Leur prévalence augmente avec l'âge et atteint son pic dans la quarantaine [I]. Toutes nos patientes avaient un âge compris entre 40 et 50 ans.

Du fait de la situation anatomique du col utérin, les léiomyomes cervicaux quand elles surviennent, sont sources de symptomatologies dominées par les métrorragies et les douleurs pelviennes [2]. Ces douleurs très souvent d'aggravation progressive sont la résultante de modifications physiques comme la pression liée à l'augmentation continue du volume du myome, les déchirures du col consécutives également à la croissance tumorale. En fonction de la taille et du sens de développement sont décrites des symptômes particuliers. Les pollakiuries et les constipations sont fréquentes en cas de léiomyomes cervicaux respectivement à développement antérieur et postérieur [7-9]. Des effets de masse sur le tractus urinaire sont rapportés dans les cas de tumeur à développement latéral responsable d'urétérohydronéphrose [7] mais aussi des présentations cliniques mimant une tumeur ovarienne [4].

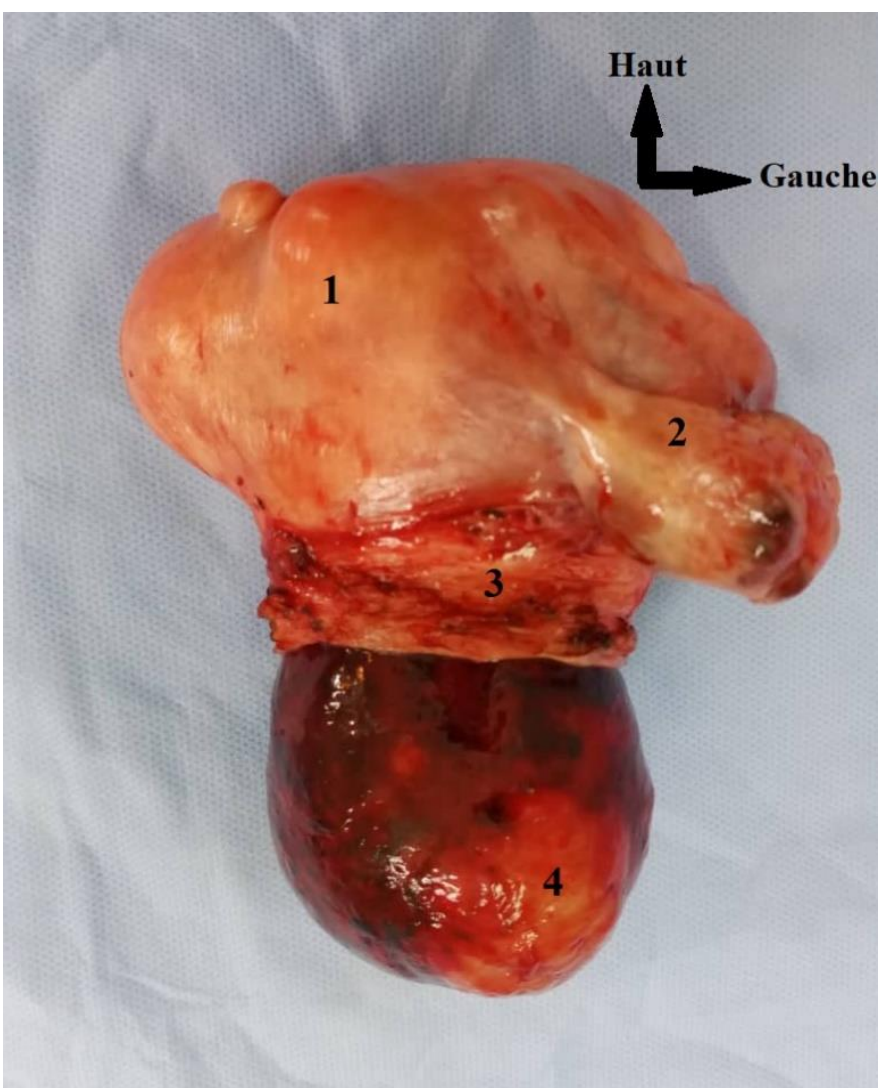

Figue 4 : Pièce opératoire d'hystérectomie totale pour myome accouché par le col. I : Corps utérin (polymyomateux), 2 : Annexe gauche, 3 : Col utérin, 4 : Myome accouché par le col.

Les lésions de petite taille sont facilement objectivées à l'examen clinique comme des tumeurs siégeant sur le col endovaginal. Dans les cas de masses abdomino-pelviennes avec développement endovaginal refoulant l'utérus vers l'abdomen, un examen sous valves pourrait permettre d'évaluer l'état des récessus cervico-vaginaux [7]. II s'agit de tumeurs fermes, à surface luisante régulièrement lisse respectant les parois vaginales telles que retrouvées dans nos observations $n^{\circ} I$ et 3 . La taille maximale retrouvée dans la littérature varie de 9 à 28 $\mathrm{cm}[\mathrm{I}, 4,7]$. Les volumineuses tumeurs au diagnostic sont courantes dans notre contexte. Elles sont corolaires des longs délais de consultation, de l'itinéraire thérapeutique particulière de nos patientes qui passent par les guérisseurs traditionnels. La persistance et l'aggravation des symptômes liés aux métrorragies dictent le recours ultime à la médecine moderne. Le diagnostic différentiel se fait d'une part avec les tumeurs isthmiques, les adénocarcinomes endocervicaux, les sarcomes 
utérins, et les tumeurs ovariennes en cas de léiomyome cervicale latérale pédiculé $[4,7]$.

Les examens radiologiques permettent d'affiner le diagnostic. L'échographie pelvienne en général et particulièrement par voie transvaginale permet de mieux explorer les myomes cervicaux [I, 7]. L'IRM est par excellence l'examen qui permet au mieux de caractériser les lésions utérines, en précisant la taille, la topographie, les nombres exacts, les contours réguliers [I]. Elle nous a permis d'évoquer fortement le diagnostic surtout dans nos cas $n^{\circ} \mathrm{I}$ et 3 .

L'exploration chirurgicale par laparotomie s'impose dans la majeure partie des cas. Elle permet dans la majorité des cas de confirmer l'origine cervico-utérine de la tumeur, et d'évaluer la disposition des organes pelviennes dans un contexte de distorsion de l'anatomie régionale [2, 4, 10]. Ainsi les possibilités thérapeutiques pourront être abordées en optimisant les risques d'incidents opératoires à type de section urétérale ou de perforation vésicale $[I, 7, I I]$. Les voies laparoscopique et transvaginale voire un bistournage sont rapportés pour des cas de myome de moins de $10 \mathrm{~cm}[1,2]$. La prise en charge des léiomyomes cervicaux est presque exclusivement chirurgicale contrairement aux localisations corporéales où un traitement médical peut être souvent entrepris [I, I0]. II peut s'agir d'hystérectomie totale comme dans notre série, où il n'y avait aucun désir de procréation ultérieure. L'examen anatomopathologique de la pièce opératoire permet de confirmer le diagnostic de léiomyome. Le pronostic est bon. Aucune complication immédiate ou à long terme n’a été notée dans notre série.

\section{CONCLUSION}

Les léiomyomes géants accouchés par le col sont rares. Les principaux symptômes demeurent les métrorragies, les douleurs pelviennes et une masse volumineuse endovaginale. L'IRM est d'un grand apport. L'hystérectomie totale constitue le traitement de choix devant des cas symptomatiques chez des patientes non désireuses de grossesse. L'évolution est favorable.

\section{CONFLITS D'INTERET}

Les auteurs n'ont déclaré aucun conflit d'intérêts.

\section{REFERENCES}

I. Vilos GA, Allaire C, Laberge PY, Layland N. Prise en charge des léiomyomes utérins. J Obstet Gynaecol Can. 2016;38(I2S):S550eS576

2. Ferrari F, Forte S, Valenti G, Ardighieri L, Barra F, Esposito V, Sartori E, Odicino F. Current treatment options for cervical léiomyomas: A systematic review of literature. Medicina 2021. 57 : 92. https://www.doi.org/ / 0.3390/medicina 57020092

3. Chalal N, Demmouche A. Profil épidémiologique des fibromes utérins dans la région de Sidi Bel Abbes, Algérie. Pan Afr Med J. 2013 ; 15 :7. Doi : https://www. I0.1 1604/pamj/.2013.15.7.2690

4. Goel N, Seth S. An unusual case of cervical fibroid masquerading as ovarian tumor. J Mid-life Health. 2016;7:144-6.

5. Tiemtoré-Kambou BMA, Baguiyan A, Lamien PD, Koama A, Napon AM, Bamouni YA, Diallo O, Cissé R. Myome, découverte fortuite ou métrorragie : qui dit mieux ? P Afr Med J. 2021;38:388.
6. Kénémé $B$, Ciss $D, K a S$, Mbaye F, Dem A, Sembène M. Uterine fibroids in senegal: polymorphism of medI 2 gene and correlation with epidemiological factors. Ame J Can Res and Rev, 2018,2:4

7. Geetha AM. Lantern on Saint Paul's dôme - A case report of cervical fibroid. Indian Journal of Obstetrics and Gynecology Research. 2021;8(1): I 10-1 I2

8. Kansu-Celik H, Evliyaoglu O, Karakaya BK, Tarlan N, Ozel S, Engin-Ustun Y. Two case of acute urinary retention caused by large cervical leiomyoma with review of litterature. J Exp Ther Oncol. 2019;। 3(I):4I-43.

9. Mihmanli V, Cetinkaya N, Kilickaya, Kilinc A, Köse D. Giant cervical myoma associated with urinary incontience and hydroureteronephosis. Clin Exp Obstet Gynecol. 2015 ; 42(5) : 690-I.

I0. Wong J, Tan GHC, Nadarajah R, et al. BMJ Case Rep 2017: [29 May 202I]. doi:I0.II 36/bcr-2017- 22I408; Consulté le 29 May 202I à 13H40. Disponible sur https://casereports.bmj.com/content/2017/bcr-2017221408.long

II. Kavitha B, Jyothi R, Rama Devi A, Madhuri K, Sachin Avinash K, Murthy SGK. A rare case of central cervical fibroid with characteristic "LANTERN ON TOP OF ST.PAUL” appearance. Int J Res Dev Health. 20। 4;2(I):45-47 